\title{
UNIVALENCE OF GAUSSIAN AND CONFLUENT HYPERGEOMETRIC FUNCTIONS
}

\author{
SANFORD S. MILLER AND PETRU T. MOCANU
}

(Communicated by Clifford J. Earle, Jr.)

\begin{abstract}
Conditions are determined for the univalence convexity and starlikeness of Gaussian and confluent hypergeometric functions. In addition, subordination results are obtained for these classes of functions.
\end{abstract}

\section{INTRODUCTION}

The surprising use of hypergeometric functions in the recent proof of the Bieberbach conjecture by L. de Branges [2] in 1985 has prompted renewed interest in these classes of functions. Prior to this proof there had been only a few articles in the literature dealing with the relationships between these special functions and univalent function theory. In a series of articles in 1959-60, E. Kreyszig and J. Todd investigated the univalence of the error function $\operatorname{Erf}(z)$ [3], the function $\exp \left(z^{2}\right) \cdot \operatorname{Erf}(z)$ [4], and the Bessel function $z^{1-v} J_{v}(z)$ [5]. In $1961 \mathrm{E}$. Merkes and W. Scott [6] investigated the starlikeness of certain Gaussian hypergeometric functions, while, in 1984, B. Carlson and D. Shaffer [1] defined a convolution operator involving an incomplete beta function and obtained results for starlike and prestarlike functions. The first sentence of the latter paper, which was submitted in 1983, is rather striking in light of de Branges' proof a year later. It reads "Connections between the theory of univalent functions and the theory of special functions have not received much attention."

In 1986 St. Ruscheweyh and V. Singh [8] investigated the order of starlikeness of certain hypergeometric functions. An important part of their proof employs a refined version of the method of continued fractions used by Merkes and Scott.

Received by the editors February 27, 1989.

1980 Mathematics Subject Classification (1985 Revision). Primary 33A30; Secondary 30C45.

Key words and phrases. Gaussian and confluent hypergeometric functions, univalent, convex, starlike, differential subordination.

The first author acknowledges support received from the International Research \& Exchange Board (IREX), with funds provided by the National Endowment for the Humanities and the United States Information Agency. 
In this paper we determine the univalency of certain hypergeometric functions by a totally different method. We employ the method of differential subordinations and obtain results different from those of Merkes and Scott, and those of Ruscheweyh and Singh. In addition, some other results involving subordination of hypergeometric functions are obtained.

In $\S 2$ we provide some definitions and a key lemma, while $\S 3$ deals with confluent hypergeometric functions and $\S 4$ deals with Gaussian hypergeometric functions.

\section{Preliminaries}

A function $f$, analytic in the unit disc $U$, is said to be convex if it is univalent and $f(U)$ is convex. It is well known that $f$ is convex if and only if $f^{\prime}(0) \neq 0$ and $\operatorname{Re}\left[z f^{\prime \prime}(z) / f^{\prime}(z)+1\right]>0$ in $U$. A function $f$ analytic in $U$, with $f(0)=0$, is said to be starlike if it is univalent and $f(U)$ is starlike with respect to the origin. The function $f$ is starlike if and only if $f(0)=0$, $f^{\prime}(0) \neq 0$ and $\operatorname{Re}\left[z f^{\prime}(z) / f(z)\right]>0$ in $U$. If in addition, $\operatorname{Re}\left[z f^{\prime}(z) / f(z)\right]>$ $\alpha, 0 \leq \alpha<1$, then $f$ is called starlike of order $\alpha$.

Since subordination is used in several of the theorems, we now restate its definition. Let $f$ and $g$ be analytic in $U$. The function $f$ is subordinate to $g$, written $f \prec g$ or $f(z) \prec g(z)$, if $g$ is univalent, $f(0)=g(0)$ and $f(U) \subset g(U)$.

We close this section with a lemma that will be used to prove several theorems in the remaining sections.

Lemma A. Let $E$ be a set in the complex plane $\mathrm{C}$ and let a function $H: \mathrm{C}^{3} \times$ $U \rightarrow \mathbf{C}$ satisfy the condition:

$$
\begin{aligned}
& H(i s, t, u+i v ; z) \notin E, \text { for } z \in U \text { and for real } s, t, u, v \\
& \text { satisfying } t \leq-\left(1+s^{2}\right) / 2 \text { and } t+u \leq 0
\end{aligned}
$$

If $p$ is analytic in $U$, with $p(0)=1$ and $H\left(p(z), z p^{\prime}(z), z^{2} p^{\prime \prime}(z) ; z\right) \in E$, $z \in U$, then $\operatorname{Re}(p(z)>0$ in $U$.

This lemma is a special case of Theorem 1 in [7] with $q(z)=(1+z) /(1-z)$. If we only have $H: \mathbf{C}^{2} \times U \rightarrow \mathbf{C}$, then condition (1) simplifies to

$$
H(i s, t ; z) \notin E \text {, for } z \in U \text { and real } s, t \text { with } t \leq-\left(1+s^{2}\right) / 2 \text {. }
$$

\section{CONFLUENT HYPERGEOMETRIC FUNCTIONS}

Let $a$ and $c$ be complex numbers with $c \neq 0,-1,-2, \ldots$, and consider the function defined by

$$
\begin{aligned}
\Phi(a ; c ; z) & ={ }_{1} F_{1}(a ; c ; z) \\
& =1+\frac{a}{c} \frac{z}{1 !}+\frac{a(a+1)}{c(c+1)} \frac{z^{2}}{2 !}+\frac{a(a+1)(a+2)}{c(c+1)(c+2)} \frac{z^{3}}{3 !}+\cdots .
\end{aligned}
$$


This function, referred to as the confluent (or Kummer) hypergeometric function, is analytic in $\mathbf{C}$ and satisfies Kummer's hypergeometric differential equation

$$
z w^{\prime \prime}(z)+[c-z] w^{\prime}(z)-a w(z)=0 .
$$

If we let $(d)_{k}=\Gamma(d+k) / \Gamma(d)=d(d+1) \cdots(d+k-1)$ and $(d)_{0}=1$, then (3) can be written in the form

$$
\Phi(a ; c ; z)=\sum_{k=0}^{\infty} \frac{(a)_{k}}{(c)_{k}} \frac{z^{k}}{k !}=\frac{\Gamma(c)}{\Gamma(a)} \sum_{k=0}^{\infty} \frac{\Gamma(a+k)}{\Gamma(c+k)} \frac{z^{k}}{k !} .
$$

We list some elementary properties of $\Phi$ that can be obtained from (5) and can be found in [9].

$$
\begin{gathered}
c \Phi^{\prime}(a ; c ; z)=a \Phi(a+1 ; c+1 ; z) \\
\Phi(a ; a ; z)=e^{z}
\end{gathered}
$$

If $\operatorname{Re} c>\operatorname{Re} a>0$ then

$$
\Phi(a ; c ; z)=\frac{\Gamma(c)}{\Gamma(a) \Gamma(c-a)} \int_{0}^{1} t^{a-1}(1-t)^{c-a-1} e^{t z} d t=\int_{0}^{1} e^{t z} d \mu(t),
$$

where $\mu(t)=[\Gamma(c) / \Gamma(a) \Gamma(c-a)] t^{a-1}(1-t)^{c-a-1}$ is a probability measure on $[0,1]$. In fact

$$
\int_{0}^{1} d \mu(t)=[\Gamma(c) / \Gamma(a) \Gamma(c-a)] \cdot B(a, c-a)=1,
$$

where $B$ is the beta function.

Our intention is to determine conditions on $a$ and $c$ so that $\Phi$ will be univalent in $U$. We first determine conditions for $\Phi$ to have positive real part in $U$.

Lemma 1. If $a$ and $c$ are real and satisfy one of the conditions:

(i) $a>0$ and $c \geq a$, or

(ii) $a \leq 0$ and $c \geq 1+\left(1+a^{2}\right)^{1 / 2}$,

then $\operatorname{Re} \Phi(a ; c ; z)>0$ for $z \in U$.

Proof. If (i) holds, then the result follows immediately from (7) and (8). Suppose next that (ii) holds and let $p(z)=\Phi(a ; c ; z)$. Since $p(z)$ satisfies (4), it will also satisfy

$$
z^{2} p^{\prime \prime}(z)+[c-z] z p^{\prime}(z)-a z p(z)=0 .
$$

If we let $H\left(w_{1}, w_{2}, w_{3} ; z\right)=w_{3}+[c-z] w_{2}-a z w_{1}$ and $E=\{0\}$, then (10) can be written as $H\left(p(z), z p^{\prime}(z), z^{2} p^{\prime \prime}(z) ; z\right) \in E$. Now use Lemma A to prove that $\operatorname{Re}(p(z)>0$. If we let $z=x+i y$ then

$$
\operatorname{Re} H(i s, t, u+i v ; x+i y)=t+u+(c-1-x) t+a y s .
$$


Since (ii) implies that $c>2$, we have $c-1-x>0$ for $z \in U$ and deduce that

$$
\begin{aligned}
\operatorname{Re} H(i s, t, u+i v ; z) & \leq-\frac{1}{2}(c-1-x)\left(1+s^{2}\right)+\text { ays } \\
& =-\frac{1}{2}\left[(c-1-x) s^{2}-2 a y s+(c-1-x)\right] \equiv Q(s),
\end{aligned}
$$

for $t \leq-\left(1+s^{2}\right) / 2$ and $t+u \leq 0$. We next show that $Q(s)<0$, for all real $s$. Condition (ii) and $x^{2}+y^{2}<1$ yield

$$
a^{2} y^{2}<\left[(c-1)^{2}-1\right]\left[1-x^{2}\right] \leq[c-1-x]^{2},
$$

and so the discriminant $D$ of $Q(s)$ satisfies

$$
D=a^{2} y^{2}-(c-1-x)^{2}<0 \text {. }
$$

Since $Q(0)<0$, from $(11)$ we obtain $\operatorname{Re} H(i s, t, u+i v ; z)<0$ for $z \in U$, $t \leq-\left(1+s^{2}\right) / 2$ and $t+u \leq 0$. Hence, by Lemma A, we conclude $\operatorname{Re} p(z)=$ $\operatorname{Re} \Phi(a ; c ; z)>0$ for $z \in U$.

We now obtain our first univalence condition for confluent hypergeometric functions. If we apply Lemma 1 to (6) we immediately obtain

Theorem 1. If $a \neq 0$ and $c$ are real and satisfy one of the following conditions

(i) $a>-1$ and $c \geq a$, or

(ii) $a \leq-1$ and $c \geq\left(1+(a+1)^{2}\right)^{1 / 2}$,

then $\operatorname{Re}(c / a) \Phi^{\prime}(a ; c ; z)>0$ for $z \in U$, and hence $\Phi(a ; c ; z)$ is univalent in $U$.

We next determine conditions for the convexity of $\Phi$.

Theorem 2. If $a \neq 0$ and $c$ are real and satisfy $c>N(a)$, where

$$
N(a)= \begin{cases}|a|+1 / 2, & \text { if }|a| \geq 1 / 3, \\ 3 a^{2} / 2+2 / 3, & \text { if }|a| \leq 1 / 3,\end{cases}
$$

then $\Phi(a ; c ; z)$ is convex in $U$.

Proof. A check of cases shows that $c>N(a)$ implies that either condition (i) or (ii) of Theorem 1 holds. Hence $\Phi^{\prime}(z)=\Phi^{\prime}(a ; c ; z) \neq 0$, and the function $p(z)=1+z \Phi^{\prime \prime}(z) / \Phi^{\prime}(z)$ is analytic in $U$ with $p(0)=1$. Since $\Phi$ satisfies the differential equation (4), using this transformation in (4) results in

$$
z p^{\prime}(z)+p^{2}(z)+(c-2-z) p(z)-a z-c+1=0 .
$$

If we let $H\left(w_{1}, w_{2} ; z\right)=w_{2}+w_{1}^{2}+(c-2-z) w_{1}-a z-c+1$ and $E=\{0\}$, then (13) can be written as $H\left(p(z), z p^{\prime}(z) ; z\right) \in E$. We will use (2) and Lemma A to prove that $\operatorname{Re} p(z)>0$. Letting $z=x+i y$ we obtain

$$
\begin{aligned}
\operatorname{Re} H(i s, t ; z) & =t-s^{2}+y s-x a-c+1 \\
& \leq-\left[3 s^{2}-2 y s+2 x a+2 c-1\right] / 2 \equiv Q(s),
\end{aligned}
$$


for $t \leq-\left(1+x^{2}\right) / 2$. We next show that the quadratic $Q(s)<0$, for all real $s$ and $x^{2}+y^{2}<1$. The discriminant $D$ of $Q(s)$ satisfies

$$
D=y^{2}-6 x a-3(2 c-1)<4-6 c-6 a x-x^{2} \equiv h(x) .
$$

If $|a| \leq 1 / 3$ then $h^{\prime}\left(x_{0}\right)=0$ for $x_{0}=-3 a$, and using (12) we obtain $h(x) \leq$ $h\left(x_{0}\right)=4-6 c+9 a^{2} \leq 0$ for $-1<x<1$. If $|a| \geq 1 / 3$ then $h(x)$ is monotone on $(-1,1)$, and from (12) we deduce $h(x)<3-6 c+6|a| \leq 0$. Hence, in both cases, $D<0$ for $x^{2}+y^{2}<1$. Using (12) again, we find that $Q(0)<0$ and from (14) deduce $\operatorname{Re} H(i s, t ; z)<0$ for $z \in U$ and all real $s, t$, with $t \leq-\left(1+s^{2}\right) / 2$. By Lemma A and (2) we conclude that $\operatorname{Re} p(z)=\operatorname{Re}\left[z \Phi^{\prime \prime}(z) / \Phi^{\prime}(z)+1\right]>0$, which shows that $\Phi$ is convex in $U$.

Corollary 2.1. If $c \geq 1+N(a-1)$, where $N(a)$ is defined by (12), then $z \Phi(a ; c ; z)$ is starlike in $U$.

Proof. From (6) we obtain

$$
(a-1) z \Phi(a ; c ; z)=(c-1) z \Phi^{\prime}(a-1 ; c-1 ; z) .
$$

If $a \neq 1$ then the result follows immediately by applying Theorem 2 . If $a=1$ the result follows by using a limit procedure.

We next use Theorem 2 to obtain a subordination result for some confluent hypergeometric functions.

Corollary 2.2. If $a \neq 0$ and $d>c>N(a)$, where $N(a)$ is defined by (12) then

$$
\Phi(a ; d ; z) \prec \Phi(a ; c ; z) \text {. }
$$

Proof. If we use (5) to represent $\Phi(a ; c ; t z)$ and multiply both sides of (5) by $t^{c-1}(1-t)^{d-c-1}$, then, after integrating on $[0,1]$, we obtain

$$
\begin{gathered}
\frac{\Gamma(c)}{\Gamma(a)} \sum_{k=0}^{\infty} \frac{\Gamma(a+k)}{\Gamma(c+k)} \frac{z^{k}}{k !} \int_{0}^{1} t^{k+c-1}(1-t)^{d-c-1} d t \\
\quad=\int_{0}^{1} \Phi(a ; c ; t z) t^{c-1}(1-t)^{d-c-1} d t .
\end{gathered}
$$

Since

$$
\begin{aligned}
\int_{0}^{1} t^{k+c-1}(1-t)^{d-c-1} d t & =B(c+k, d-c) \\
& =\Gamma(c+k) \Gamma(d-c) / \Gamma(d+k),
\end{aligned}
$$

where $B$ is the beta function, (16) simplifies to

$$
\frac{\Gamma(c) \Gamma(d-c)}{\Gamma(a)} \sum_{k=0}^{\infty} \frac{\Gamma(a+k)}{\Gamma(d+k)} \frac{z^{k}}{k !}=\int_{0}^{1} \Phi(a ; c ; t z) t^{c-1}(1-t)^{d-c-1} d t
$$

Using this in (5),

$$
\begin{aligned}
\Phi(a ; d ; z) & =\frac{\Gamma(d)}{\Gamma(c) \Gamma(d-c)} \int_{0}^{1} \Phi(a ; c: t z) t^{c-1}(1-t)^{d-c-1} d t \\
& =\int_{0}^{1} \Phi(a ; c ; t z) d \mu(t)
\end{aligned}
$$


where $\mu$ is a probability measure as in (9). From (17) we conclude, from a well-known formula, that $\Phi(a ; d ; z)$ is contained in the convex hull of $\{\Phi(a ; d ; t z) \mid t \in[0,1], z \in U\}$. However, by Theorem $2, \Phi(a ; d ; z)$ is a convex function, and thus (15) holds.

Example 1. If $a=1$ and $c=\delta+1$ then, by (8) and Theorem 2, the function

$$
f_{\delta}(z)=\Phi(1 ; \delta+1 ; z)=\delta \int_{0}^{1}(1-t)^{\delta-1} e^{t z} d t
$$

is convex in $U$ if $\delta \geq 1 / 2$. In addition, by Corollary 2.2 we have $f_{\eta} \prec f_{\delta}$ if $\eta>\delta>1 / 2$. (Note that the original series (5) implies $f_{\delta}(z) \prec f_{0}(z)=e^{z}$ ). By Corollary 2.1 the function $z f_{\delta}(z)$ is starlike in $U$ for $\delta \geq 2 / 3$. Since $z f_{\delta+1}(z)=(\delta+1)\left[f_{\delta}(z)-1\right]$, we deduce that $f_{\delta}(z)-1$ is starlike in $U$, for $\delta>-1 / 3$.

We next determine conditions on $a$ and $c$ so that $z \Phi(a ; c ; z)$ will be starlike of order $1 / 2$.

Theorem 3. If $a$ and $c$ are real and satisfy $c \geq R(a)$ where

$$
R(a)= \begin{cases}2 a & \text { if } 3 / 4 \leq a, \\ (1-2 a)^{2}+5 / 4 & \text { if } 1 / 4 \leq a<3 / 4, \\ 2(1-a) & \text { if } a<1 / 4,\end{cases}
$$

then the function $z \Phi(a ; c ; z)$ is starlike of order $1 / 2$ in $U$.

Proof. The technique is similar to that of the proof of Theorem 2 and only the highlights will be mentioned. Let $g(z)=z \Phi(a ; c ; z)$. Since $c \geq R(a)$ implies that either condition (i) or (ii) of Lemma 1 holds, we deduce that $g(z) / z \neq 0$ in $U$. If we set

$$
p(z)=2 z g^{\prime}(z) / g(z)-1,
$$

then $p(z)$ is analytic in $U$ with $p(0)=1$. Since $g(z) / z$ satisfies the differential equation (4), if we apply the transformation (19) in (4) we obtain a first order differential equation of the form

$$
H\left(p(z), z p^{\prime}(z) ; z\right)=0 .
$$

Applying condition (18) leads to $\operatorname{Re} H(i s, t ; z)<0$ for $z \in U$ and real $s$, $t$ with $t \leq-\left(1+s^{2}\right) / 2$. Hence, by Lemma A we obtain $\operatorname{Re} p(z)>0$, which shows that $g=z \Phi(a ; c ; z)$ is starlike of order $1 / 2$.

Example 2. If $\delta \geq 1$, then the function

$$
g_{\delta}(z)=z \Phi(1 ; \delta+1 ; z)=\delta z \int_{0}^{1}(1-t)^{\delta-1} e^{t z} d t
$$

is starlike of order $1 / 2$. 
Corollary 3.1. If $c \geq R(a)$, where $R(a)$ is given by (18) then $z \Phi\left(a ; c ; z^{2}\right)$ is starlike in $U$.

Proof. If we let $h(z)=z \Phi\left(a ; c ; z^{2}\right)$ then $h(z)=g\left(z^{2}\right) / z$, where $g(z)=$ $z \Phi(a ; c ; z)$. Since $g$ is starlike of order $1 / 2$ and

$$
\frac{z h^{\prime}(z)}{h(z)}=\frac{2 z^{2} g^{\prime}\left(z^{2}\right)}{g\left(z^{2}\right)}-1,
$$

we conclude that $h$ is starlike in $U$.

Example 3. If $a=1 / 2$ in the last corollary, then the function

$$
z \Phi\left(1 / 2 ; c ; z^{2}\right)=\frac{z \Gamma(c)}{\sqrt{\pi} \Gamma(c-1 / 2)} \int_{0}^{1} t^{-1 / 2}(1-t)^{c-3 / 2} e^{t z^{2}} d t
$$

is starlike for $c \geq 5 / 4$. In the special case when $c=3 / 2$, if we replace $z$ with $i z$ and simplify then

$$
z \Phi\left(1 / 2 ; 3 / 2 ;-z^{2}\right)=\int_{0}^{z} e^{-w^{2}} d w=\operatorname{Erf}(z)
$$

is starlike in $U$.

\section{GAUSSIAN HYPERGEOMETRIC FUNCTIONS}

Let $a, b$ and $c$ be complex numbers with $c \neq 0,-1,-2, \ldots$ The function

$$
F(a, b ; c ; z)={ }_{2} F_{1}(a ; b ; c ; z)=1+\frac{a b}{c} \frac{z}{1 !}+\frac{a(a+1) b(b+1)}{c(c+1)} \frac{z^{2}}{2 !}+\cdots,
$$

called the (Gaussian) hypergeometric function, is analytic in $U$ and satisfies the hypergeometric differential equation

$$
z(1-z) w^{\prime \prime}+[c-(a+b+1) z] w^{\prime}-a b w=0 .
$$

Using the notation introduced in $\S 3$ we can rewrite $F$ as

$$
F(a, b ; c ; z)=\sum_{k=0}^{\infty} \frac{(a)_{k}(b)_{k}}{(c)_{k}} \frac{z^{k}}{k !}=\frac{\Gamma(c)}{\Gamma(a) \Gamma(b)} \sum_{k=0}^{\infty} \frac{\Gamma(a+k) \Gamma(b+k)}{\Gamma(c+k)} \frac{z^{k}}{k !} .
$$

We list some elementary properties of $F$ that can be obtained from (21) and can be found in [8]:

$$
\begin{gathered}
F(a, b ; c ; z)=F(b, a ; c ; z), \\
c F^{\prime}(a, b ; c ; z)=a b F(a+1, b+1 ; c+1 ; z) \\
F(a, b ; c ; z)=(1-z)^{c-a-b} F(c-a, c-b ; c ; z), \\
F(a, b ; b ; z)=(1-z)^{-a} .
\end{gathered}
$$

If $\operatorname{Re} c>\operatorname{Re} b>0$ then there is a probability measure on $[0,1]$ such that

$$
F(a, b ; c ; z)=\int_{0}^{1}(1-t z)^{-a} d \mu(t) .
$$


Because of the symmetry in (22), any condition we determine involving $a$ and $b$ will have an analog involving $b$ and $a$. We will not list these analogs.

The following lemma, which will be needed to prove the main theorem of this section, provides conditions for the local univalency of hypergeometric functions.

Lemma 2. If $a, b, c$ are real and satisfy

$$
-1 \leq b \leq c \text { and } a \in[-2,0) \cup[c-1, c+1] \text {, }
$$

then $F^{\prime}(a, b ; c ; z) \neq 0$ for $z \in U$.

Proof.

Case 1. $-1<b<c$ and $a \in[-2,0)$. By employing (23) and (26) we obtain

$$
F^{\prime}(a, b ; c ; z)=a b \int_{0}^{1}(1-t z)^{-(a+1)} d \mu(t) \text {. }
$$

Since $a \in[-2,0)$ we have $\operatorname{Re}(1-t z)^{-(a+1)}>0$ for $t \in[0,1]$ and $z \in U$. Using this result in the above equation we obtain $F^{\prime}(a, b ; c ; z) \neq 0$ for $z \in U$.

Case 2. $-1<b<c$ and $a \in[c-1, c+1]$. From (23) (24) and (26) we have

$$
F^{\prime}(a, b ; c ; z)=a b(1-z)^{c-a-b-1} \int_{0}^{1}(1-t z)^{-(c-a)} d \mu(t) .
$$

Since $|c-a| \leq 1, \operatorname{Re}(1-t z)^{-(c-a)}>0$ and again $F^{\prime}(a, b ; c ; z) \neq 0$ for $z \in U$.

Case 3. If $b=c$, from (24) we obtain $F(a, b ; b ; z)=(1-z)^{a}$. If $b=-1$, from $(21)$ then $F(a,-1 ; c ; z)=1-a z / c$. In both of these cases $F^{\prime} \neq 0$.

Theorem 4. If $a, b, c$ are real and satisfy $-2 \leq a<0,-1 \leq b, b \neq 0$ and $c>M(a, b)$, where

$$
M(a, b)=\max \{2+|a+b|, 1-a b\},
$$

then $F(z)=F(a, b ; c ; z)$ is convex in $U$.

Proof. From the conditions on $a$ and $b$ it is easy to show that $b \leq 2+|a+b| \leq$ $M(a, b)$. Hence $c>b$ and, from Lemma $2, F^{\prime}(z) \neq 0$ in $U$. If we set $p(z)=1+z F^{\prime \prime}(z) / F^{\prime}(z)$, then $p(z)$ is analytic in $U$ and satisfies $p(0)=1$. If we use this substitution in the hypergeometric differential equation (20), then $p$ will satisfy the following first order differential equation

$$
(1-z) z p^{\prime}(z)+(1-z) p^{2}(z)+\{c-2-(a+b) z\} p(z)+1-c-a b z=0 .
$$

Since $c>M(a, b)$ implies $c-2-(a+b) z \neq 0$ in $U$, we can rewrite (28) in the form

$$
J(z)\left[z p^{\prime}(z)+p^{2}(z)\right]+p(z)+[J(z)-K(z)] / 2=0
$$


where $J(z)=(1-z) /(c-2-(a+b) z)$ and $K(z)=(2 c-1-(1-2 a b) z) /$ $(c-2-(a+b) z)$.

A bilinear transformation $W(z)=(1+A z) /(1+B z)$, with $-1 \leq A \leq 1$ and $-1 \leq B \leq 1$, has the property that $\operatorname{Re} W(z)>0$ for $z \in U$. The conditions on $a, b$, and $c$ given in the hypothesis imply that $J(z)$ and $K(z)$ are such bilinear transforms. Hence we have

$$
\operatorname{Re} J(z)>0 \text { and } \operatorname{Re} K(z)>0 \text { for } z \in U .
$$

If we let $E=\{0\}$ and define the function

$$
H\left(w_{1}, w_{2} ; z\right)=J(z) \cdot\left[w_{2}+w_{1}^{2}\right]+w_{1}+[J(z)-K(z)] / 2,
$$

then (29) becomes $H\left(p(z), z p^{\prime}(z) ; z\right) \in E$. By using (30) we obtain

$$
\begin{aligned}
\operatorname{Re} H(i s, t ; z) & =\left(t-s^{2}+1 / 2\right) \operatorname{Re} J(z)-\operatorname{Re} K(z) / 2 \\
& \leq\left(-\left(1+s^{2}\right) / 2-s^{2}+1 / 2\right) \operatorname{Re} J(z)-\operatorname{Re} K(z) / 2<0,
\end{aligned}
$$

for $z \in U$ and real $s, t$ with $t \leq\left(1+s^{2}\right) / 2$. Hence $H$ satisfies (2), and by Lemma A we deduce $\operatorname{Re}(p(z)>0$, which shows that $F$ is convex in $U$.

Example 1. If we take $a=-2, b=1$ and $c \geq 3$, then all conditions of the theorem are satisfied. Hence

$$
F(-2,1 ; c, z)=1-\frac{2}{c} z+\frac{2}{c(c+1)} z^{2}
$$

is convex in $U$. This simple example cannot be handled by the conditions of Merkes and Scott [6] or of Ruscheweyh and Singh [8].

Corollary 4.1. If $a, b, c$ are real and satisfy $-1 \leq a \leq 1,0 \leq b$, and $c>1+M(a-1, b-1)$, where $M(a, b)$ is defined by $(27)$, then $z F(a, b ; c ; z)$ is starlike in $U$.

Proof. From (23) we obtain

$$
(a-1)(b-1) z F(a, b ; c ; z)=(c-1) z F^{\prime}(a-1, b-1 ; c-1 ; z) .
$$

If $a \neq 1$ and $b \neq 1$, then, by Theorem $1, F(a-1, b-1 ; c-1 ; z)$ will be convex and the conclusion follows by logarithmically differentiating (31). If $a=1$ or $b=1$ the conclusion follows by a limit procedure.

Remark. The incomplete beta function is defined in terms of the Gaussian hypergeometric function by

$$
\phi(a, c ; z)=z F(a, 1 ; c ; z)=z+\sum_{k=1}^{\infty} \frac{(a)_{k}}{(c)_{k}} z^{k+1} .
$$

In light of the last corollary, $\phi(a, c ; z)$ will be starlike if $-1 \leq a \leq 1$ and $c>3+|a-1|$. 
Corollary 4.2. If $-2 \leq a<0,-1 \leq b, b \neq 0$ and $d>c>M(a, b)=$ $\max \{2+|a+b|, 1-a b\}$ then

$$
F(a, b ; d ; z) \prec F(a, b ; c ; z) .
$$

Proof. By a technique similar to that in Corollary 2.2 we obtain

$$
F(a, b ; d ; z)=\frac{\Gamma(d)}{\Gamma(c) \Gamma(d-c)} \int_{0}^{1} F(a, b ; c ; t z) t^{c-1}(1-t)^{d-c-1} d t
$$

or

$$
F(a, b ; d ; z)=\int_{0}^{1} F(a, b ; c ; t z) d \mu(t),
$$

where $\mu$ is a probability measure. By Theorem $4 F(a, b ; c ; z)$ is convex, and, using this in the above equation, we obtain the desired subordination.

\section{REFERENCES}

1. B. C. Carlson and D. B. Shaffer, Starlike and prestarlike hypergeometric functions, SIAM J. Math. Anal. 15 (1984), 737-745.

2. L. De Branges, $A$ proof of the Bieberbach conjecture, Acta Math. 154 (1985), 137-152.

3. E. Kreyszig and J. Todd, The radius of univalence of the error function, Numer. Math. 1 (1959), 78-89.

4. - On the radius of univalence of the function $\exp \left(z^{2}\right) \int_{0}^{2} \exp \left(-t^{2}\right) d t$, Pacific J. Math. 9 (1959), 123-127.

5. _ The radius of univalence of Bessel functions, Illinois J. Math. 4 (1960), 143-149.

6. E. P. Merkes and W. T. Scott, Starlike hypergeometric functions, Proc. Amer. Math. Soc. 12 (1961), 885-888.

7. S. S. Miller and P. T. Mocanu, Differential subordinations and inequalities in the complex plane, J. Differential Equations 67 (1987), 199-211.

8. St. Ruscheweyh and V. Singh, On the order of starlikeness of hypergeometric functions, J. Math. Anal. Appl. 113 (1986), 1-11.

9. G. Sansone and J. Gerretsen, Lectures on the theory of functions of a complex variable, Vol. II, Wolters-Noordhoof Publishing, Groningen, The Netherlands, 1969.

Department of Mathematics, State University of New York, Brockport, New York 14420

Department of Mathematics, University of Cluj-Napoca, 3400 Cluj-Napoca, Romania 